\title{
Spin constraints on nuclear energy density functionals
}

\author{
L.M. Robledd* \\ Departamento de Física Teórica, Módulo 15, \\ Universidad Autónoma de Madrid, E-28049 Madrid, Spain \\ R.N. Bernard \\ Departamento de Física Atómica, Molecular y Nuclear, \\ Universidad de Granada, E-18071, Granada, Spain \\ G.F. Bertsch \\ Institute for Nuclear Theory and Dept. of Physics, Box 351560, \\ University of Washington, Seattle, Washington 98915, USA
}

\begin{abstract}
Abstract: The Gallagher-Moszkowski rule in the spectroscopy of odd-odd nuclei imposes a new spin constraint on the energy functionals for self-consistent mean field theory. The commonly used parameterization of the effective three-body interaction in the Gogny and Skyrme families of energy functionals is ill-suited to satisfy the spin constraint. In particular, the Gogny parameterization of the three-body interaction has the opposite spin dependence to that required by the observed spectra. The two-body part has a correct sign, but in combination the rule is violated as often as not. We conclude that a new functional form is needed for the effective three-body interaction that can take into better account the different spin-isospin channels of the interaction.
\end{abstract}

*Electronic address: luis.robledo@uam.es; URL: http://gamma.ft.uam.es/robledo 
Nuclear energy density functionals have reached a mature state with respect to the parameterization of time-even fields required for the Hartree-Fock-Bogoliubov theory of ground state properties of even-even nuclei. A theory encompassing nuclei with odd numbers of nucleons requires a good understanding of the time-odd fields as well. Some aspects of the time-odd fields have been examined in the literature [1 5], but important experimental information has been ignored in determining the parameters in the functionals. In particular, the spin dependence of the neutron-proton interaction is crucial to determine ground-state spins of odd-odd nuclei. We will show in this letter that an energy functional from the Gogny family of functionals strongly violates an empirical rule for determine ground state spins. The Gogny functional has a very specific form for an effective three-body interaction which automatically has the wrong sign for the spin dependence. The other leading family of functionals, based on Skyrme's parameterization, has the same form for spatial dependence of the effective three-body interaction, and is likely to have the similar difficulties. Indeed, it was shown long ago that the contact parameterization could lead to instabilities in nuclear Hartree-Fock theory[1].

The rule that should be respected was formulated by Gallagher and Moszkowski (GM) [6] for the quasiparticle angular momentum couplings in strongly deformed odd-odd nuclei. Under those conditions the components of the angular momentum $K_{p}, K_{n}$ of the odd nucleons about the symmetry axis are good quantum numbers. The two possible relative spin orientations, $K_{p}+K_{n}$ and $\left|K_{p}-K_{n}\right|$ give rise to two separate rotational bands having band-head angular momentum $J=\left|K_{p} \pm K_{n}\right|$. According to the rule [6, 7], the oriention with parallel intrinsic spins is the lower energy band. As documented in a review of the GM rule [7], there are only rare exceptions to the rule.

We have developed new code to find the Hartree-Fock-Bogoliubov minima of the Gogny functional in axially symmetric nuclei, treating for the first time time-odd fields including the spin-dependent ones [8]. Applying the code to spin splittings in deformed nuclei, we found that the predicted splittings violated the GM rule more often than not. In retrospect, the result is not too surprising because as stated earlier none of the energy functionals in common use have been fitted to spin-dependent properties[19].

We now examine the origin of the results. It is useful to distinguish the two-particle interaction and the three-particle interaction present in the functionals. In principle there are enough degrees of freedom in the parameterization of the two-particle interaction to 
take into account the GM splittings. However, the three-particle interaction is essential for nuclear saturation and, for computational simplicity, it has a very constrained parameterization. Namely, it is a density-dependent contact interaction in both the Skyrme and Gogny functionals of the form

$$
t_{3}\left(1+x \hat{P}_{\sigma}\right) \delta\left(\vec{r}_{1}-\vec{r}_{2}\right) \rho\left(\left(\vec{r}_{1}+\vec{r}_{2}\right) / 2\right)^{\alpha}
$$

in the standard notation[10]. It is further restricted to the parallel-spin interaction $(x=-1)$ in the Gogny functionals. It must be repulsive to saturate nuclear matter, but it can't have a significant antiparallel-spin component because that channel requires an attractive interaction overall to produce BCS pairing.

We now illustrate the problem with a well-known example, the nucleus ${ }^{174} \mathrm{Lu}$. The odd nucleons in ground band have angular momenta and parities $\left(K_{p}, K_{n}\right)=\left(7 / 2^{+}, 5 / 2^{-}\right)$for the proton and neutron respectively. These correspond to Nilsson orbitals [404] $\downarrow_{p}$ and [512] $\uparrow_{n}$. The spins are parallel for antiparallel orbital angular momentum, i.e. $K=\left|K_{p}-K_{n}\right|$. Indeed, the ground state band has $K^{\pi}=1^{-}$in agreement with the Gallagher-Moszkowski rule. The other coupling of angular momenta, $K=K_{p}+K_{n}=6^{-}$, is associated with an excited band with a band head at $171 \mathrm{keV}$ excitation. The experimental levels are compared with the HFB calculations in Fig. 1. We first show the spectra of neighboring odd-A nuclei on the lefthand and middle panels. In the middle one, the theory confirmed the ground band assignment of a quasiparticle in the [512] $\uparrow_{n}$ Nilsson orbital. However, the theory does not predict the correct ordering of the proton quasiparticle energies, shown in the left-hand panel. As a consequence, the [404] $\downarrow_{p}[512] \uparrow_{n}$ appear as excited states in the theoretical spectrum of the ${ }^{174} \mathrm{Lu}$, shown in the right-hand panel. One sees that the level ordering is opposite to the experimental, with the $6^{-}$band head below the $1^{-}$, thus violating the GM rule[20].

To understand to theoretical splittings in more detail, we separate three contributions: 1) the spin dependence of the two-body interaction, treating the interaction in first-order perturbation theory 2) the spin dependence of the density-dependent interaction, again treating it perturbatively 3) the many-body rearrangement effects associated with the wave function modifications in the two-quasiparticle state. The three contributions are +188 and $-291 \mathrm{keV}$ for the two-particle and three-particle perturbative contributions, respectively. The rearrange contribution is $+44 \mathrm{keV}$, giving a total splitting of $-61 \mathrm{keV}$ as shown in the level scheme in Fig. 1. This should be compared with an empirical value of +114 , which is 


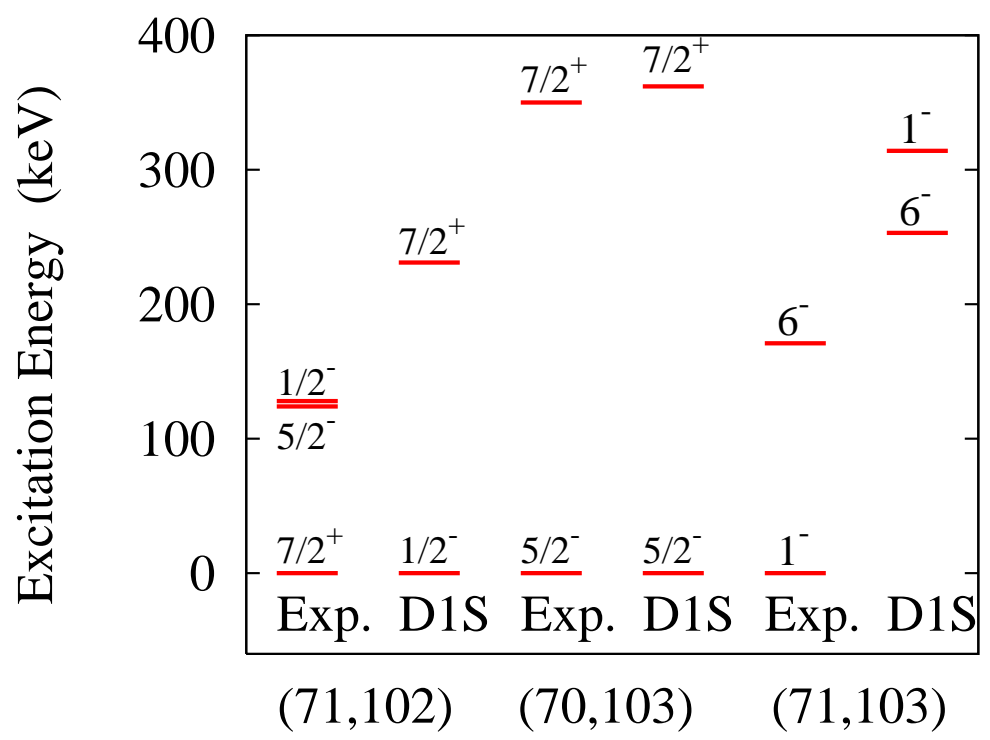

Figure 1: Low-lying band heads in the spectra of the nucleus ${ }^{174} \mathrm{Lu}$ and odd-A neighbors: ${ }^{173} \mathrm{Lu}$ (left); ${ }^{173} \mathrm{Yb}$ (center) ${ }^{174} \mathrm{Lu}$ (right). Due to the inversion of the lowest proton quasiparticle energies, the ground-state doublet in ${ }^{174} \mathrm{Lu}$ is not the lowest two-quasiparticle configuration in the calculated spectrum. Lower energy calculated configurations are not shown.

what is left of the observed splitting of +171 after the rotational effects have been removed [7]. Thus, as claimed earlier, the three-particle contribution has a bad sign and here it even overwhelms the good sign of the two-particle contribution.

To see how general these findings are, we have performed this analysis on 100-225 doublets in each of 15 nuclei in the deformed rare-earth region. All of these nuclei have strong prolate deformations. The results are shown in Table I. These results confirm the statements made earlier that: the two-body interaction has a correct sign; the three-body interaction has the wrong sign; and the net sign with all the contributions is variable and inconsistent with a general GM rule.

To gain a better understanding of the origin of the problem we briefly review how the interaction energies are calculated using the one-body densities of Hartree-Fock-Bogoliubov (HFB) theory. When time-reversal symmetry is broken the one body-density matrix can be decomposed as the sum of a time-even and a time-odd density. In the expression of the total energy there is a contribution which is quadratic in the time-odd term. Starting from an even-even HFB reference state, the blocking of a given quasiparticle leads to a non-zero time odd density matrix. The blocking of the time reversed state leads to the 


\begin{tabular}{|c|ccc|c|}
\hline \hline & $2 \mathrm{BP}$ & $3 \mathrm{BP}$ & $\mathrm{FP}$ & self-consistent \\
\hline $164-168 \mathrm{Ho}$ & $93 \%$ & $8 \%$ & $28 \%$ & $45 \%$ \\
$168-172 \mathrm{Tm}$ & 97 & 4 & 26 & 41 \\
$172-176 \mathrm{Lu}$ & 97 & 4 & 28 & 40 \\
$180-184 \mathrm{Ta}$ & 97 & 5 & 37 & 30 \\
$184-188 \mathrm{Lu}$ & 97 & 3 & 36 & 28 \\
\hline
\end{tabular}

Table I: Theoretical spin splittings of neutron-proton configurations for odd-odd nuclei in the rare earth region. For each nucleus, two-quasiparticle states were constructed taking 10 to 15 proton quasiparticle orbitals and a like number of neutron quasiparticle orbitals. The table shows the percentage of the cases in which the calculated splitting agrees with the GM rule, combining the results for several isotopes of each element. Columns labeled 2BP, 3BP, and FP show the perturbative results for the two-body interaction alone, the three-body interaction alone, and the full interaction treated perturbatively. The last column shows the results of the fully self-consistent calculation of the HFB minima. The table shows the results for the D1S interaction. We also have calculated splittings with the D1M interactions [11] and found similar results.

same time odd density but with opposite sign. To build the two configurations defining a GM pair a proton quasiparticle with quantum number $K_{p}$ and a neutron one with $K_{n}$ are blocked to obtain one of the states. The other is obtained by blocking $\left(-K_{p}, K_{n}\right)$. In this way both the spin parallel and antiparallel configurations are considered[21]. The time-odd proton densities of both configurations are the same in absolute value but have opposite signs whereas the two time odd neutron densities are the same. From these considerations it becomes clear that only those terms of the energy depending on the product of a time odd proton density times a time odd neutron one are contributing to the energy splitting of the doublet. Among the different terms contributing to the energy in the Gogny interaction there are a few that do not contribute to the splitting, namely the Wigner term of the central potential, the Coulomb potential and the pairing channel of the central potential. Among the remaining terms, the spin-orbit contribution is much smaller than the other two and will be omitted in the discussion. Therefore the splitting of the doublet is dominated by the central two-body and three-body contributions. We calculate the perturbative contribution 
to the splitting starting a wave function at the HFB minimum of an even-even nucleus. The required quasiparticles are then blocked and the expection value of the energy is calculated. As an example, the three-body contribution to the splitting is given by

$$
\begin{gathered}
\Delta E=E(\uparrow, \uparrow)-E(\downarrow, \uparrow) \\
=4 t_{3} \int d^{3} \vec{r} \rho^{\alpha}\left(\rho_{1 / 2,1 / 2}^{p, o d d} \rho_{1 / 2,1 / 2}^{n, \text { odd }}+\bar{\rho}_{1 / 2,-1 / 2}^{p, \text { odd }} \bar{\rho}_{1 / 2,-1 / 2}^{n, \text { odd }}\right)
\end{gathered}
$$

where $\rho$ is the ordinary density, a function of $\vec{r}$ alone. The needed time-odd component of the density matrix $\rho_{s, s^{\prime}}^{t, \text { odd }}$ depends on nucleon type $t$ and spin projection $s, s^{\prime}$ as well. The bar denotes the modulus of a (complex) density. If the blocked quasiparticle is BCS-like (i.e. linear combinations of creation and annihilation canonical basis states) then the timeodd density $\rho_{s, s^{\prime}}^{t, \text { odd }}$ is diagonal in the canonical basis with zeroes in the diagonal except for the blocked orbital quantum number where it takes the value $\pm 1 / 2$ depending on whether the spin $\sigma$ of the blocked orbital points up or down. In this very specific case only taking place at the first iteration (first order) the density $\bar{\rho}_{1 / 2,-1 / 2}^{t, \text { odd }}$ is zero and $\rho_{1 / 2,1 / 2}^{t, \text { odd }}$ equals $\sum_{q}\left|\varphi_{t, q}(\vec{r})\right|^{2} / 2(-1)^{\sigma-1 / 2}$. Therefore $\Delta E$ as defined above is positive for parallel spins and negative for antiparallel ones, just the opposite of the GM rule. In the actual HFB calculation the blocked quasiparticle may have a mixture of the two spin orientations and the simple argument given above may fail. This occurs for some configurations treated in the Table.

It is also of interest to examine the various interactions in a momentum space representation. The relevant plane-wave matrix elements of the two-body Gogny interaction are given by

$$
\begin{gathered}
<q|V| q>_{\uparrow \uparrow}^{n p}=\pi^{3 / 2} \sum_{i=1}^{2} \mu_{i}^{3}\left(W_{i}+B_{i}+\left(H_{i}+M_{i}\right) e^{-\left(q \mu_{i}\right)^{2}}\right) \\
<q|V| q>_{\uparrow \downarrow}^{n p}=\pi^{3 / 2} \sum_{i=1}^{2} \mu_{i}^{3}\left(W_{i}+M_{i} e^{-\left(q \mu_{i}\right)^{2}}\right)
\end{gathered}
$$

in the notation of Ref. [12]. The momentum $q=\left|k_{n}-k_{p}\right| / 2$ is the relative momentum of the two nucleons.

We now make a qualitative connection with the energy difference for the two spin couplings of nucleons, changing the momentum of the proton at the same time as its spin is flipped. The difference is 22 ]

$$
\Delta v^{2 b}=\pi^{3 / 2} \sum_{i=1}^{2} \mu_{i}^{3}\left(B_{i}+\left(H_{i}+M_{i}\right) e^{-\left(q_{p} \mu_{i}\right)^{2}}-M_{i} e^{-\left(q_{\alpha} \mu_{i}\right)^{2}}\right)
$$



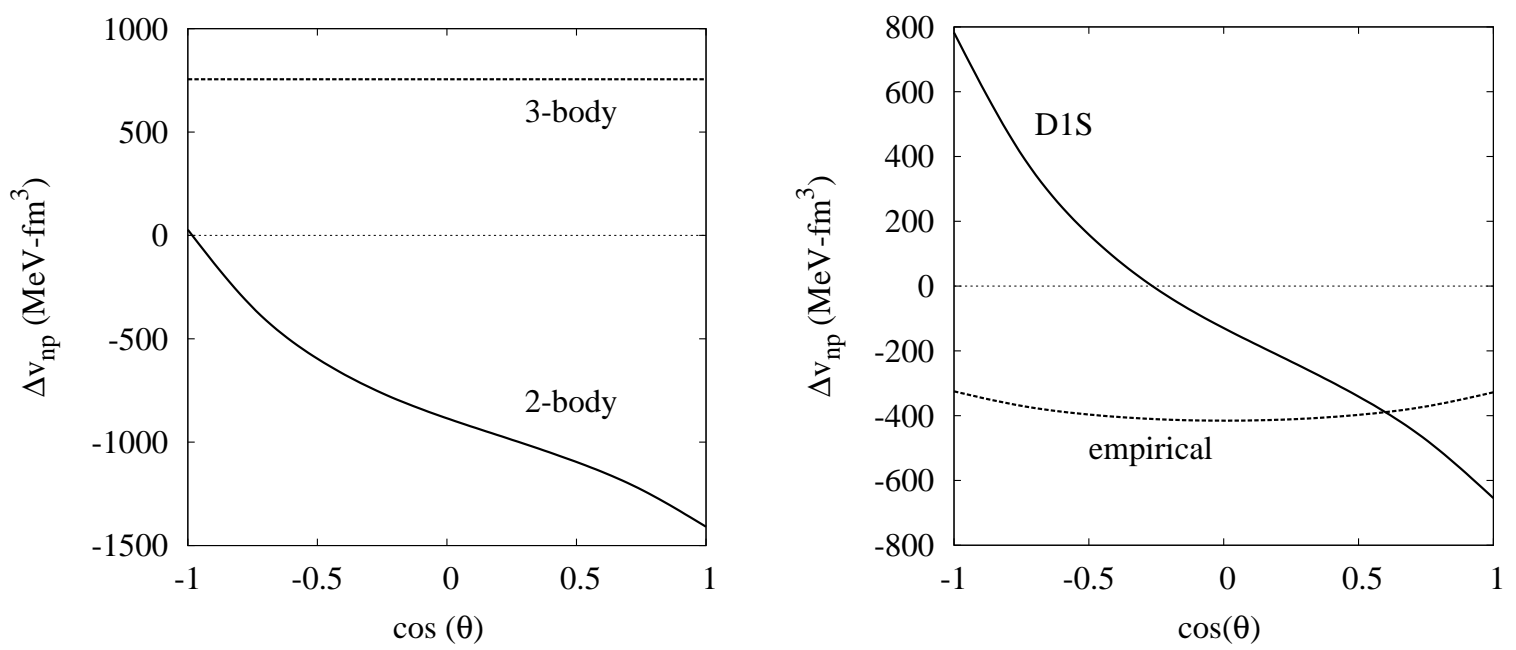

Figure 2: Matrix elements of the effective neutron-proton interaction from the D1S Gogny energy functional at nuclear matter density. In the left-hand panel, the individual contributions of the two- and three-body terms from Eq. (3) and (4) are shown. In the right-hand panel the total for the D1S is shown in comparison to the empirical $\Delta v_{n p}$ discussed in Refs. [7, 13].

with $q_{p}, q_{a}=\left(k_{n}^{2}+k_{p}^{2} \mp 2 k_{p} k_{n} \cos \theta\right)^{1 / 2} / 2$ and $\cos \theta=\vec{k}_{n} \cdot \vec{k}_{p} / k_{n} k_{p}$. The corresponding difference of matrix elements for the three-body interaction is independent of $q$ and is given by

$$
\Delta v^{3 b}=t_{3} \rho^{\alpha}
$$

These differences for nucleons on the Fermi surface are plotted in Fig. 2 as a function of $\cos \theta$, taking the Fermi momentum as $k_{f}=1.35 \mathrm{fm}^{-1}$. In the left-hand panel one see that the two components have opposite sign. They are added together in the plot on the right. Here one can see that both signs are present, depending on the angle $\theta$. In that plot we also show an empirical neutron-proton interaction, constructed to fit the data on the GM multiplets. The interactions are in rough agreement when the momenta in the parallel-spin state are also parallel, but strongly disagree when the momenta are anti-parallel in that state.

We conclude with some remarks the construction of a better three-body interaction. It seems clear that the contact nature of the Gogny (and Skyrme) interactions is at the root of the problem of reproducing the empirical spin-dependence of the neutron-proton interaction. There have been proposals in the literature to generalize the three-body interaction by including derivative terms [14, 15] as in the Skyrme two-body interaction. Unfortunately 
the expansion in powers of the derivatives gives rise to many terms and it is difficult from a purely empirical point of view to determine the coefficients. The interaction arises both from the subnucleon degrees of freedom that are missing from theory as well as from the correlations that are missing from the mean-field treatment of the nucleon degrees of freedom. The latter, called the induced three-body interaction, has a long range [16] and a nonlocality [17] that is impossible to take account of in a contact interaction. A finite range has to be introduced in some fashion, but the computational cost is very high. Besides the Skyrme approach using derivatives of contact interactions, it may be possible to reduce the computational cost of finite-range three-body interactions using hypercontraction[18] or separable parameterizations. We feel it would be very worthwhile to develop codes that could apply them

\section{ACKNOWLEDGMENTS}

We thank J. Dobaczewski for comments. The work of GFB was supported by the U.S. Department of Energy under Grant DE-FG02-00ER41132, and by the National Science Foundation under Grant PHY-0835543. The work of LMR was supported by MICINN grants Nos. FPA2012-34694, FIS2012-34479 and by the Consolider-Ingenio 2010 program MULTIDARK CSD2009-00064.

[1] K-H. Passler, Nucl. Phys. A257 2531976.

[2] U. Post, E. Wuest, and U. Mosel, Nucl. Phys. A437 274 (1985).

[3] J. Dobaczewski and J. Dudek, Phys. Rev. C 1827 (1995).

[4] M. Bender, J. Dobaczewski, J. Engel and W. Nazarewicz, Phys. Rev. C 65054322 (2002).

[5] K.J. Pototzky, J. Erler, and P.-G. Reinhard et al., Eur. Phys. J. A 46299 (2010).

[6] C. Gallagher and S. Moszkowski, Phys. Rev. 1111282 (1958).

[7] J. Boisson, R. Piepenbring, and W. Ogle, Phys. Rep. 2699 (1976).

[8] L.M. Robledo, R. Bernard, and G.F. Bertsch, Phys. Rev. C 86064313 (2012).

[9] M. Kortelainen and T. Lesinski, J. Phys. G 37064039 (2010).

[10] M. Bender, P.-H. Heenen, and P.-G. Reinhard, Rev. Mod. Phys. 75121 (2003). 
[11] S. Goriely, S. Hilaire, M. Girod, and S. Perú, Phys. Rev. Lett. 102, 242501 (2009).

[12] J.F. Berger, M. Girod, and D. Gogny, Comp. Phys. Comm. 63365 (1991).

[13] A. Covello, A. Gargano, and N. Itaco, Phys. Rev. C 563092 (1997).

[14] F. Raimondi, B.G. Carlsson, and J. Dobaczewski, Phys. Rev. C 83054311 (2011).

[15] J. Sadoudi, T. Duguet, and J. Meyer et al., arXiv:1310.0854 (2013).

[16] A. Gezerlis and G.F. Bertsch, Phys. Rev. Lett. 105212501 (2010).

[17] A. Gezerlis and G.F. Bertsch, Phys. Rev. C 85037303 (2012).

[18] R. Parrish, E. Hohenstein, N. Schuck, et al., Phys. Rev. Lett. 111132505 (2013).

[19] There has been some cognizance of the spin properties when it was realized that some parameterizations lead to disasterous instabilities [9].

[20] The splittings has additional contributions besides the spin-dependent interactions. However, these are smaller [7] and generally do not change the sign of the splitting.

[21] Which one is which depends on the decomposition of $K$ into orbital and spin components, $K=\ell_{z}+s_{z}$.

[22] There is another spin-isospin combination of the interaction term corresponding to the Landau parameters for the interaction[4, App. E]. It is quite different from Eq. (5) . 